\title{
Magic Numbers in the Discrete Tomography of Cyclotomic Model Sets
}

\author{
Christian Huck
}

\begin{abstract}
We report recent progress in the problem of distinguishing convex subsets of cyclotomic model sets $\Lambda$ by (discrete parallel) X-rays in prescribed $\Lambda$-directions. It turns out that for any of these model sets $\Lambda$ there exists a 'magic number' $m_{\Lambda}$ such that any two convex subsets of $\Lambda$ can be distinguished by their X-rays in any set of $m_{\Lambda}$ prescribed $\Lambda$-directions. In particular, for pentagonal, octagonal, decagonal and dodecagonal model sets, the least possible numbers are in that very order $11,9,11$ and 13 .
\end{abstract}

\section{Introduction}

Discrete tomography is concerned with the inverse problem of retrieving information about some finite set in Euclidean space from (generally noisy) information about its slices. One important problem is the unique reconstruction of a finite point set in Euclidean 3-space from its (discrete parallel) $X$-rays in a small number of directions, where the $X$-ray of the finite set in a certain direction is the line sum function giving the number of points in the set on each line parallel to this direction.

The interest in the discrete tomography of planar Delone sets $\Lambda$ with long-range order is motivated by the requirement in materials science for the unique reconstruction of solid state materials like quasicrystals slice by slice from their images under quantitative high resolution transmission electron microscopy (HRTEM). In fact, in [12], [14] a technique is described, which can, for certain crystals, effectively measure the number of atoms lying on densely occupied columns. Clearly, the aforementioned density condition forces us to consider only $\Lambda$-directions, i.e. directions parallel to lines through two different points of $\Lambda$.

Christian Huck

Fakultät für Mathematik, Universität Bielefeld, Postfach 100131, 33501 Bielefeld, Germany, email: huck@math.uni-bielefeld.de 
In the quasicrystallographic setting, the positions to be determined form a finite subset of a nonperiodic Delone set with long-range order (more precisely, a model set [2], [13]). Model sets possess a dimensional hierarchy, i.e. they allow a slicing into planar model sets. In fact, many of the model sets that describe real quasicrystallographic structures allow a slicing such that each slice is an $n$-cyclotomic model set, the latter being (planar) Delone sets contained in the additive subgroup of the Euclidean plane generated by the $n$th roots of unity; cf. [6], [7], [9] and [15] for details. It therefore suffices to study the discrete tomography of these cyclotomic model sets. In practice, the cases $n=5,8,12$ are of particular interest. In the present text, we shall mainly focus on the larger class of cyclotomic Delone sets.

Since different finite subsets of an $n$-cyclotomic model set $\Lambda$ may have the same $\mathrm{X}$-rays in several directions, one is naturally interested in conditions to be imposed on the set of directions together with restrictions on the possible finite subsets of $\Lambda$ such that the latter phenomenon cannot occur. Here, we consider the convex subsets of $\Lambda$ and summarise recent results in the problem of distinguishing convex subsets of $\Lambda$ by X-rays in prescribed $\Lambda$-directions. It turns that there are four prescribed $\Lambda$ directions such that any two convex subsets of $\Lambda$ can be distinguished by the corresponding X-rays, whereas less than four $\Lambda$-directions never suffice for this purpose. Much more novel is the result obtained in collaboration with M. Spieß that there is a finite number $m_{\Lambda}$ such that any two convex subsets of $\Lambda$ can be distinguished by their X-rays in any set of $m_{\Lambda}$ prescribed $\Lambda$-directions. Moreover, the least possible numbers $m_{\Lambda}$ in the case of the practically most relevant examples of $n$-cyclotomic model sets $\Lambda$ with $n=5,8$ and 12 only depend on $n$ and are in that very order 11,9 and 13. This extends a well-known result of R. J. Gardner and P. Gritzmann [5] on the corresponding problem for the crystallographic cases $n=3,4$ of the triangular resp. square lattice $\Lambda$ (with least possible number $m_{\Lambda}=7$ in both cases) to cases that are relevant in quasicrystallography.

The intention of this text is to provide an easy to read guide to the results of [10] with a view towards practical applications. Detailed proofs, related results and an extensive list of references can be found there. For the algorithmic reconstruction problem in the quasicrystallographic setting, the reader is referred to [3], [7].

\section{Cyclotomic Delone sets}

Throughout the text, the Euclidean plane is identified with the complex numbers. For $z \in \mathbb{C}, \bar{z}$ denotes the complex conjugate of $z$. Further, we denote by $K_{\Lambda}$ the smallest subfield of $\mathbb{C}$ that contains the rational numbers as well as the union of $\Lambda-\Lambda$ and its image $\overline{\Lambda-\Lambda}$ under complex conjugation. Recall that $\Lambda$ is called a Delone set if it is both uniformly discrete and relatively dense. For $n \in \mathbb{N}$, we always let $\zeta_{n}=e^{2 \pi i / n}$, a primitive $n$th root of unity in $\mathbb{C}$. Then, the smallest subfield of $\mathbb{C}$ that contains the rational numbers as well as $\zeta_{n}$ is the $n$th cyclotomic field denoted by $\mathbb{Q}\left(\zeta_{n}\right)$. The latter ist just the $\mathbb{Q}$-span of the $n$th roots of unity and thus contains the $\mathbb{Z}$-span $\mathbb{Z}\left[\zeta_{n}\right]$ of the $n$th roots of unity. Recall that a homothety of the complex plane is given by 
$z \mapsto \lambda z+t$, where $\lambda \in \mathbb{R}$ is positive and $t \in \mathbb{C}$. For the purpose of this text, the following rather abstract definition provides a convenient framework.

Definition 1. Let $n \geq 3$. A Delone set $\Lambda \subset \mathbb{C}$ is called an $n$-cyclotomic Delone set if it satisfies the following properties:

(n-Cyc) $K_{\Lambda}$ is contained in $\mathbb{Q}\left(\zeta_{n}\right)$.

(Hom) For any finite subset $F$ of $K_{\Lambda}$, there is a homothety $h$ of the complex plane that maps the elements of $F$ to $\Lambda$.

Further, $\Lambda$ is called a cyclotomic Delone set if it is an $n$-cyclotomic Delone set for a suitable $n \geq 3$.

Standard examples of $n$-cyclotomic Delone sets are the $n$-cyclotomic model sets, which were also called cyclotomic model sets with underlying $\mathbb{Z}$-module $\mathbb{Z}\left[\zeta_{n}\right]$ in $[9$, Section 4.5] and are defined via the canonical cut and project scheme that is given by the Minkowski representation of the $\mathbb{Z}$-module $\mathbb{Z}\left[\zeta_{n}\right]$; see [9, 10] for details. These sets are certain Delone subsets of the $\mathbb{Z}$-module $\mathbb{Z}\left[\zeta_{n}\right]$ and range from periodic examples like the fourfold square lattice $(n=4)$ or the sixfold triangular lattice $(n=3)$ to nonperiodic examples like the vertex set of the tenfold Tübingen triangle tiling $(n=5)$, the eightfold Ammann-Beenker tiling $(n=8)$ or the twelvefold shield tiling $(n=12)$; see [8, Figure 1], [9, Figure 2] and Figure[1 below for illustrations. Note that the vertex sets of the famous Penrose tilings of the plane fail to be 5cyclotomic model sets but can still be seen to be 5-cyclotomic Delone sets; see [1] and references therein.

\section{Determination of convex subsets by $\mathrm{X}$-rays}

Let $\left(t_{1}, t_{2}, t_{3}, t_{4}\right)$ be an ordered tuple of four distinct elements of $\mathbb{R} \cup\{\infty\}$. Then, its cross ratio $\left\langle t_{1}, t_{2}, t_{3}, t_{4}\right\rangle$ is the nonzero real number defined by

$$
\left\langle t_{1}, t_{2}, t_{3}, t_{4}\right\rangle=\frac{\left(t_{3}-t_{1}\right)\left(t_{4}-t_{2}\right)}{\left(t_{3}-t_{2}\right)\left(t_{4}-t_{1}\right)},
$$

with the usual conventions if one of the $t_{i}$ equals $\infty$.

The unit circle in $\mathbb{C}$ is denoted by $\mathbb{S}^{1}$ and its elements are also called directions. For a nonzero complex number $z$, we denote by $\operatorname{sl}(z)$ the slope of $z$, i.e. $\operatorname{sl}(z)=$ $-i(z-\bar{z}) /(z+\bar{z}) \in \mathbb{R} \cup\{\infty\}$. Let $\Lambda$ be a subset of $\mathbb{C}$. A direction $u \in \mathbb{S}^{1}$ is called a $\Lambda$-direction if it is parallel to a nonzero element of the difference set $\Lambda-\Lambda=$ $\{v-w \mid v, w \in \Lambda\}$ of $\Lambda$. By construction, the cross ratio of slopes of four pairwise nonparallel $\Lambda$-directions is an element of the field $K_{\Lambda} \cap \mathbb{R}$. In case of $n$-cyclotomic Delone sets $\Lambda$, these cross ratios are thus elements of the field $\mathbb{Q}\left(\zeta_{n}\right) \cap \mathbb{R}$.

Definition 2. $\bullet$ Let $F$ be a finite subset of $\mathbb{C}$, let $u \in \mathbb{S}^{1}$ be a direction, and let $\mathscr{L}_{u}$ be the set of lines in the complex plane in direction $u$. Then the (discrete parallel) 
$X$-ray of $F$ in direction $u$ is the function $X_{u} F: \mathscr{L}_{u} \rightarrow \mathbb{N}_{0}=\mathbb{N} \cup\{0\}$, defined by

$$
X_{u} F(\ell)=\operatorname{card}(F \cap \ell)
$$

- Let $\mathscr{F}$ be a collection of finite subsets of $\mathbb{C}$ and let $U \subset \mathbb{S}^{1}$ be a finite set of directions. We say that the elements of $\mathscr{F}$ are determined by the X-rays in the directions of $U$ if different elements of $\mathscr{F}$ cannot have the same X-rays in the directions of $U$.

One can easily see that no finite set of pairwise nonparallel $\Lambda$-directions suffices in order to determine the whole class of finite subsets of $\Lambda$ by the corresponding $\mathrm{X}$-rays [9]. It is therefore necessary to impose some restriction on the finite subsets of $\Lambda$ to be determined. It has proven most fruitful to focus on the convex subsets of cyclotomic Delone sets. The latter are bounded (and thus finite) subsets $C$ of $\Lambda$ satisfying the equation $C=\operatorname{conv}(C) \cap \Lambda$, where $\operatorname{conv}(C)$ denotes the convex hull of $C$. One has the following fundamental result which shows that one has to choose the set $U$ of $\Lambda$-directions in such a way that certain convex polygons cannot exist; cf. [9. Proposition 4.6 and Lemma 4.5]. Here, for a finite set $U \subset \mathbb{S}^{1}$ of directions, a nondegenerate convex polygon $P \subset \mathbb{C}$ is called a $U$-polygon if it has the property that whenever $v$ is a vertex of $P$ and $u \in U$, the line in the complex plane in direction $u$ which passes through $v$ also meets another vertex $v^{\prime}$ of $P$. $P$ is called a $U$-polygon in $\Lambda$, if its vertices lie in $\Lambda$. Note that the proof of direction (ii) $\Rightarrow$ (i) needs property (Hom) and see Figure 1 for an illustration of the other direction (i) $\Rightarrow$ (ii).

Theorem 1. Let $\Lambda$ be a cyclotomic Delone set and let $U \subset \mathbb{S}^{1}$ be a set of two or more pairwise nonparallel $\Lambda$-directions. The following statements are equivalent:

(i) The convex subsets of $\Lambda$ are determined by the $X$-rays in the directions of $U$.

(ii) There is no $U$-polygon in $\Lambda$.

In addition, if $\operatorname{card}(U)<4$, then there is a $U$-polygon in $\Lambda$.

Employing Darboux's theorem on second midpoint polygons [4] together with a blend of sophisticated methods from the theory of cyclotomic fields and previous results obtained by Gardner and Gritzmann [5], one obtains the following deep result on $U$-polygons; cf. [10, Theorem 5.7]. Note that the proof heavily relies on property (n-Cyc).

Theorem 2. Let $n \geq 3$ and let $\Lambda$ be an $n$-cyclotomic Delone set. Further, let $U \subset \mathbb{S}^{1}$ be a set offour or more pairwise nonparallel $\Lambda$-directions and suppose the existence of a $U$-polygon. Then the cross ratio of slopes of any four directions of $U$, arranged in order of increasing angle with the positive real axis, is an element of the finite set $\mathscr{C}_{\mathrm{lcm}(2 n, 12)}\left(\mathbb{Q}\left(\zeta_{n}\right) \cap \mathbb{R}\right)$ of numbers in the field $\mathbb{Q}\left(\zeta_{n}\right) \cap \mathbb{R}$ that can be written in the form

$$
\frac{\left(1-\zeta_{\operatorname{lcm}(2 n, 12)}^{k_{1}}\right)\left(1-\zeta_{\operatorname{lcm}(2 n, 12)}^{k_{2}}\right)}{\left(1-\zeta_{\operatorname{lcm}(2 n, 12)}^{k_{3}}\right)\left(1-\zeta_{\operatorname{lcm}(2 n, 12)}^{k_{4}}\right)}
$$




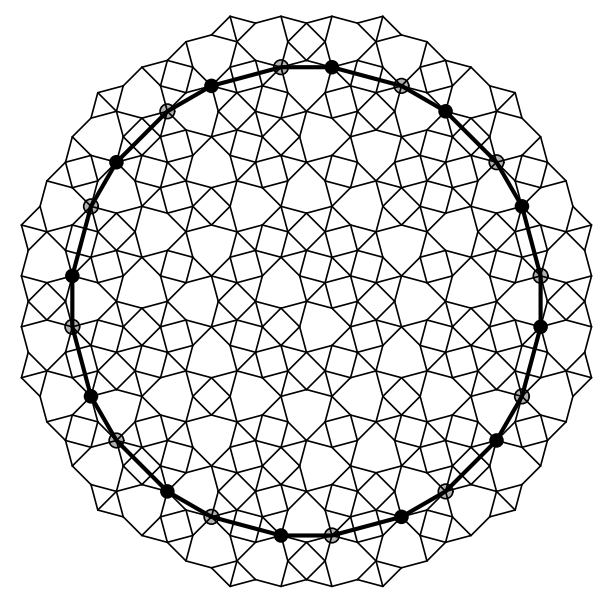

Fig. 1 The boundary of a $U$-polygon in the vertex set $\Lambda$ of the twelvefold shield tiling, where $U$ is the set of twelve pairwise nonparallel $\Lambda$-directions given by the edges and diagonals of the central regular dodecagon. The vertices of $\Lambda$ in the interior of the $U$-polygon together with the vertices indicated by the black and grey dots, respectively, give two different convex subsets of $\Lambda$ with the same X-rays in the directions of $U$.

where $\left(k_{1}, k_{2}, k_{3}, k_{4}\right)$ is an element of the set

$$
\left\{\left(k_{1}, k_{2}, k_{3}, k_{4}\right) \in \mathbb{N}^{4} \mid k_{3}<k_{1} \leq k_{2}<k_{4} \leq \operatorname{lcm}(2 n, 12)-1 \text { and } k_{1}+k_{2}=k_{3}+k_{4}\right\} \text {. }
$$

Moreover, $\operatorname{card}(U)$ is bounded above by a finite number $b_{n} \in \mathbb{N}$ that only depends on $n$. In particular, one can choose $b_{3}=b_{4}=6, b_{5}=10, b_{8}=8$ and $b_{12}=12$.

Theorems 1 and 2 now immediately imply our main result on the determination of convex subsets of cyclotomic Delone sets; cf. [10, Theorem 5.11].

Theorem 3. Let $n \geq 3$ and let $\Lambda$ be an $n$-cyclotomic Delone set.

(a) There are sets of four pairwise nonparallel $\Lambda$-directions such that the convex subsets of $\Lambda$ are determined by the corresponding $X$-rays. In addition, less than four pairwise nonparallel $\Lambda$-directions never suffice for this purpose.

(b) There is a finite number $m_{n} \in \mathbb{N}$ that only depends on $n$ such that the convex subsets of $\Lambda$ are determined by the $X$-rays in any set of $m_{n}$ pairwise nonparallel $\Lambda$-directions. In particular, one can choose $m_{3}=m_{4}=7, m_{5}=11, m_{8}=9$ and $m_{12}=13$.

By Theorems 1 and 2 above, it suffices for Part (a) to take any set of four pairwise nonparallel $\Lambda$-directions such that the cross ratio of their slopes, arranged in order of increasing angle with the positive real axis, is not an element of the finite set $\mathscr{C}_{\operatorname{lcm}(2 n, 12)}\left(\mathbb{Q}\left(\zeta_{n}\right) \cap \mathbb{R}\right)$; cf. [10, Corollary 4.10] for concrete results in the practically most important cases $n=5,8,12$ of quasicrystallography. 


\section{Concluding remarks}

Our above analysis heavily relies on the assumption of ideal data and is therefore only a very first step towards a satisfactory tool for materials science. Further, it would certainly be interesting to abandon the slice by slice approach and work, for a Delone set $\Lambda$ in Euclidean 3-space, with $\Lambda$-directions in general position instead; compare the approach to $3 \mathrm{D}$ reconstruction of atomic arrangements presented in [11]. In that case, it might well be that seven is a universal magic number for the determination of convex subsets by X-rays; cf. [4, Problem 2.1].

Acknowledgements This work was supported by the German Research Council (Deutsche Forschungsgemeinschaft), within the CRC 701.

\section{References}

1. Baake, M., Huck, C.: Discrete tomography of Penrose model sets. Philos. Mag. 87, 28392846 (2007); arXiv:math-ph/0610056

2. Baake, M., Moody, R.V. (eds.): Directions in Mathematical Quasicrystals. CRM Monograph Series, vol. 13, AMS, Providence, RI (2000)

3. Baake, M., Gritzmann, P., Huck, C., Langfeld, B., Lord, K.: Discrete tomography of planar model sets. Acta Crystallogr. A 62, 419-433 (2006); arXiv:math/0609393

4. Gardner, R.J.: Geometric Tomography. 2nd edn., Cambridge University Press, New York (2006)

5. Gardner, R.J., Gritzmann, P.: Discrete tomography: determination of finite sets by X-rays. Trans. Amer. Math. Soc. 349, 2271-2295 (1997)

6. Huck, C: Discrete Tomography of Delone Sets with Long-Range Order. Ph.D. thesis (Universität Bielefeld), Logos Verlag, Berlin (2007)

7. Huck, C.: Discrete tomography of icosahedral model sets. Acta Crystallogr. A 65, 240-248 (2009); arXiv:0705.3005

8. Huck, C.: On the existence of $U$-polygons of class $c \geq 4$ in planar point sets. Discrete Math. 309, 4977-4981 (2009); arXiv:0811.3546

9. Huck, C.: Uniqueness in discrete tomography of Delone sets with long-range order. Discrete Comput. Geom. 42, no. 4, 740-758 (2009); arXiv:0711.4525

10. Huck, C., Spieß, M.: Solution of a uniqueness problem in the discrete tomography of algebraic Delone sets. J. Reine Angew. Math. (Crelle), in press; arXiv:1101.4149

11. Ishibashi, Y., Sugiura, H., Saitoh, K., Tanaka, N.: Three-dimensional reconstruction of the atomic arrangement of icosahedral quasicrystals by binary discrete tomography. Philos. Mag. 91, 2519-2527 (2011)

12. Kisielowski, C., Schwander, P., Baumann, F.H., Seibt, M., Kim, Y., Ourmazd, A.: An approach to quantitative high-resolution transmission electron microscopy of crystalline materials. Ultramicroscopy 58, 131-155 (1995)

13. Moody, R.V.: Model sets: a survey. In: Axel, F., Dénoyer, F., Gazeau, J.-P. (eds.) From Quasicrystals to More Complex Systems, pp. 145-166. EDP Sciences, Les Ulis, and Springer, Berlin (2000); arXiv:math/0002020

14. Schwander, P., Kisielowski, C., Seibt, M., Baumann, F.H., Kim, Y., Ourmazd, A.: Mapping projected potential, interfacial roughness, and composition in general crystalline solids by quantitative transmission electron microscopy. Phys. Rev. Lett. 71, 4150-4153 (1993)

15. Steurer, W.: Twenty years of structure research on quasicrystals. Part I. Pentagonal, octagonal, decagonal and dodecagonal quasicrystals. Z. Kristallogr. 219, 391-446 (2004) 